\title{
INITIATORS AND TRIGGERING CONDITIONS FOR ADAPTIVE AUTOMATION IN ADVANCED SMALL MODULAR REACTORS
}

\section{ASME 2014 Small Modular Reactors Symposium}

Katya L Le Blanc and Johanna H Oxstrand

The INL is a

U.S. Department of Energy

National Laboratory

operated by

Battelle Energy Alliance

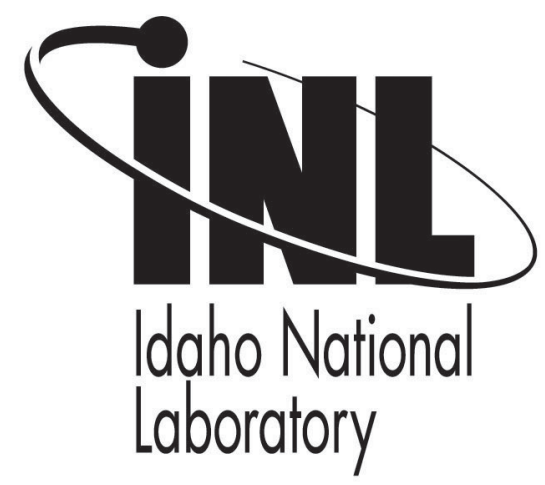

\section{April 2014}

This is a preprint of a paper intended for publication in a journal or proceedings. Since changes may be made before publication, this preprint should not be cited or reproduced without permission of the author. This document was prepared as an account of work sponsored by an agency of the United States Government. Neither the United States Government nor any agency thereof, or any of their employees, makes any warranty, expressed or implied, or assumes any legal liability or responsibility for any third party's use, or the results of such use, of any information, apparatus, product or process disclosed in this report, or represents that its use by such third party would not infringe privately owned rights. The views expressed in this paper are not necessarily those of the United States Government or the sponsoring agency. 


\section{INITIATORS AND TRIGGERING CONDITIONS FOR ADAPTIVE AUTOMATION IN ADVANCED SMALL MODULAR REACTORS}

\author{
Katya L Le Blanc \\ Idaho National Laboratory \\ Idaho Falls, Idaho, United States
}

\author{
Johanna H Oxstrand \\ Idaho National Laboratory \\ Idaho Falls, Idaho, United states
}

\section{ABSTRACT}

It is anticipated that Advanced Small Modular Reactors (AdvSMRs) will employ high degrees of automation. High levels of automation can enhance system performance, but often at the cost of reduced human performance. Automation can lead to human out-of the loop issues, unbalanced workload, complacency, and other problems if it is not designed properly. Researchers have proposed adaptive automation (defined as dynamic or flexible allocation of functions) as a way to get the benefits of higher levels of automation without the human performance costs. Adaptive automation has the potential to balance operator workload and enhance operator situation awareness by allocating functions to the operators in a way that is sensitive to overall workload and capabilities at the time of operation. However, there still a number of questions regarding how to effectively design adaptive automation to achieve that potential. One of those questions is related to how to initiate (or trigger) a shift in automation in order to provide maximal sensitivity to operator needs without introducing undesirable consequences (such as unpredictable mode changes). Several triggering mechanisms for shifts in adaptive automation have been proposed including: operator initiated, critical events, performance-based, physiological measurement, model-based, and hybrid methods. As part of a larger project to develop design guidance for human-automation collaboration in AdvSMRs, researchers at Idaho National Laboratory have investigated the effectiveness and applicability of each of these triggering mechanisms in the context of AdvSMR. Researchers reviewed the empirical literature on adaptive automation and assessed each triggering mechanism based on the human-system performance consequences of employing that mechanism. Researchers also assessed the practicality and feasibility of using the mechanism in the context of an AdvSMR control room. Results indicate that there are tradeoffs associated with each mechanism, but that some are more applicable to the
AdvSMR domain than others. The two mechanisms that consistently improve performance in laboratory studies are operator initiated adaptive automation based on hierarchical task delegation and the Electroencephalogram (EEG) -based measure of engagement. Current EEG methods are intrusive and require intensive analysis; therefore it is not recommended for an AdvSMR control rooms at this time. Researchers also discuss limitations in the existing empirical literature and make recommendations for further research.

Keywords: Adaptive Automation, Advanced Small Modular Reactors, Automation

\section{INTRODUCTION}

It is anticipated that Advanced Small Modular Reactors (AdvSMRs) will employ high degrees of automation. High levels of automation can enhance system performance, but often at the cost of reduced human performance. Automation can lead to human out-of the loop issues, unbalanced workload, complacency, and other problems if it is not designed properly [1-3]. Researchers have proposed adaptive automation (defined as dynamic or flexible allocation of functions) as a way to achieve the benefits of higher levels of automation without introducing human performance costs. Adaptive automation has the potential to balance operator workload and enhance operator situation awareness by allocating functions to the operators in a way that is sensitive to overall workload and capabilities at the time of operation. However, there still a number of questions regarding how to effectively design adaptive automation to achieve that potential. One of those questions is related to how to initiate (or trigger) a shift in automation in order to provide maximal sensitivity to operator needs without introducing undesirable consequences (such as unpredictable automation mode changes). Several triggering mechanisms for shifts in adaptive automation have been 
proposed including: operator initiated, critical events, performance based, physiological measurement, model based, and hybrid methods [4].

Although the concept of adaptive automation has been studied extensively, there is no consensus as to which triggering conditions should be used and under what conditions they should be used. Further, the appropriate triggering conditions are likely to be different depending on the operational environment. In order to assess the applicability of adaptive automation, and to identify the appropriate triggering mechanisms for AdvSMR, the existing research needs to be evaluated in the context of the AdvSMR control room. The purpose of this research is to identify the triggering mechanisms that:

1. Provide the best support for operator and system performance

2. Are likely to be achievable in the advSMR control room context

\section{METHOD}

The research team identified relevant literature by using the following search terms: adaptive automation, flexible function allocation, adaptive allocation, dynamic allocation, and adaptable automation.

The researchers then selected the articles that described empirical research investigating adaptive automation using at least one of the triggering conditions listed above to be included in this study. The researchers focused the review on addressing the following questions: What influence do the initiators have on performance? Are there any tradeoffs associated with using the initiator (e.g.., reduced workload under some conditions but increased workload under others)? What is the feasibility of using the initiator in the context of an AdvSMR control room?

Most AdvSMRs are still in the conceptual design phase, so it is difficult to fully define the operational context of the control room. However, there is a general consensus that most AdvSMR's will be highly automated [5], and that the human operator's role is likely to be to monitor the automation and only intervene during unanticipated events. Another important contextual factor to consider in AdvSMR is that many of the control rooms have not been designed yet. Therefore, even if a particular triggering condition does not seem well suited to existing nuclear power plant control rooms, the AdvSMR control room could be designed with a particular triggering condition if there is sufficient evidence that it enhances performance.

\section{RESULTS}

\subsection{Operator initiated}

Operator-initiated adaptive automation (also known as adaptable automation), allows the operator the decision authority to switch to a higher or lower level of automation. Operators may prefer this type of adaptive automation because it gives them a greater degree of control [6] than adaptive automation that switches without operator consent. However, there is evidence that performance using this triggering condition is not optimal. Sauer, Nickel, and Wastell [6] compared performance using adaptable automation on a simulated process control task to three static levels of automation (manual, decision aiding, and operation by consent). They found that the adaptable automation condition produced the worst performance in every situation except when the automation failed (in which case, performance was roughly equal to that of operation by consent). The authors concluded that under conditions of adaptable automation, operators chose to use lower levels of automation, thus compromising overall system performance.

Similarly, Kaber and Riley [7] evaluated performance using adaptive automation that was triggered based on performance on a secondary task. In one condition, the computer generated a suggestion to change the level of automation (i.e., to a higher level or lower level of automation), but the operators were not required to follow the suggestion. In the other condition, the computer-generated switch was mandatory. While both adaptive automation conditions enhanced performance compared to manual control, participants in the mandated adaptive automation condition performed better than those on the non-mandated condition. Further, secondary task performance was also lower in the non-mandated conditions, which the authors interpreted as an effect of increased workload caused by the need to evaluate the computer-generated suggestions. This study did not compare non-mandated adaptive automation to static automation, so it is difficult to draw general conclusions on how non-mandatory automation influences performance compared to other levels of static automation.

Conversely, Kidwell et al. [8] found that operatorinitiated adaptable automation enhanced change detection performance compared to a performance-based adaptive automation. The adaptable automation also enhanced operator confidence in decision making. However, the adaptable automation also increased workload. This study did not compare adaptive and adaptable automation to static conditions, so the conclusions that can be drawn from it are limited.

While traditional approaches to operator-initiated adaptive automation typically define adaptability as shifting the 
level of automation (i.e., how much of the task is automated), several researchers have advocated for designing adaptive automation based on a hierarchical task delegation method [912]. In this adaptive automation scheme, the operator still has final decision authority over shifts in automation; however the functions are broken into predetermined tasks that the operator can choose to delegate to automation. Researchers argue that this automation scheme is analogous to the way human supervisors of teams delegate tasks, and that this type of adaptable automation may be ideal for managing tradeoffs between operator workload and system unpredictability [9].

Several studies that have investigated adaptable automation based on task-delegation interfaces have found that this type of operator-initiated adaptive automation enhances performance [10-12]. Studies have also found that performance is highest when the delegation interface includes the highest level of abstraction (analogous to level of automation in this scheme), even when it is not appropriate for the current context. In two separate studies, participants executed scenarios in which the operators had to revert to fully manual performance (the lowest level of abstraction in the task delegation interface), however their performance was still enhanced by having the higher level of abstraction available [11-12]. The researchers argued that this adaptive automation scheme allows operators to use high levels of automation (thus, reducing workload) without becoming complacent [11].

One of the benefits of operator-initiated adaptive automation is that it is relatively straightforward to implement. The decision of when to shift to automation is made by the operator in real-time; therefore it does not need to be made in the design phase. Unfortunately, the fact that the operator needs to make the decision of when to automate may contribute to increased workload [9]. Additionally, when given the choice, operators may prefer to use lower levels of automation, even when it hinders system performance [6]. Recent research on adaptable automation using hierarchical task delegation is promising, and may provide a method to effectively implement adaptive automation that leaves authority in the operator's hands without compromising system performance. Additional research is needed to understand how the task delegation interface would be designed in the context of AdvSMR, and how the functions and tasks would be abstracted into differing levels. Researchers also need to demonstrate that this type of automation scheme can be successfully applied to a complex process control task such as AdvSMR.

\subsection{Critical events}

Critical event triggering may be the most straightforward way to initiate adaptive automation [13]. Despite the practicality and potentially straightforward application, the use of critical events as a triggering condition for adaptive automation has received some criticism because it is not necessarily sensitive to overall operator workload [13]. However some researchers argue that in time-critical fault situations, automation should be triggered because the human does not have the time to respond [14]. In a study investigating human operator's ability to respond to time-critical faults, Moray, Inagaki, and Itoh [14] found that adaptive automation triggered by critical events enhanced performance during severe accidents.

Although there hasn't been much research on the use of critical events as a triggering condition for adaptive automation, it should be used in time-critical situations [14]. Critical events can be implemented along with other adaptive automation schemes in AdvSMRs.

\subsection{Operator performance measurement}

Several studies have found that adaptive automation triggered by operator performance (on a primary or secondary task) enhances performance compared to manual control [15, 16, 7]. However, the fact that performance-based adaptive automation enhances performance compared to manual control does not make a strong case for performance-based adaptive automation because automation, in general, enhances system performance (unless there is an automation failure). In order to accurately assess the utility of performance-based adaptive automation, researchers need to demonstrate that it enhances performance compared to varying levels of static automation. A couple of studies have found that performance-based adaptive automation can enhance performance compared to static automation [17, 18]. Finally, one study found that performancebased adaptive automation using a change detection task also enhanced situation awareness compared to static automation [18].

Though several studies have found that performancebased adaptive automation enhances performance compared to manual performance and static automation, two studies have compared performance-based initiators with other triggering conditions and found that performance based initiators are less effective than other types of initiators. One study compared two methods for triggering adaptive automation: performance and heart rate variability (HRV). The authors found that performance was best under adaptive automation triggered by HRV [19]. Another study compared performance-based adaptive automation with operator-initiated adaptive automation, and found that participants under operator-initiated adaptive automation performed better than under performancebased adaptive automation [8].

The results of studies investigating performance-based adaptive automation are somewhat inconsistent. Furthermore, adaptive automation based on performance measurement requires that the operator have a frequent or routine task for which performance can be measured. This is not necessarily 
feasible in a supervisory control environment where the operator has little or no overt tasks assigned to him. If AdvSMR are to be highly automated it is unlikely that performance-based adaptive automation will be a feasible triggering condition. Another potential disadvantage of performance-based adaptive automation is that a shift in automation typically occurs after the system has detected a decline in performance, which has been described as initiating the adaptation after it is needed [20].

\subsection{Operator physiological assessment}

There are a variety of ways to assess operator state (e.g., workload, engagement, and fatigue) using physiological measurements. The most common physiological methods used to assess operator state for the purposes of initiating adaptive automation are Electroencepholagram (EEG) and Electrocardiogram (ECG). EEG is typically used as a way to measure operator engagement $[21,22]$ ECG is typically used as a way to measure workload, and the most common method is using heart rate variability (HRV).

Some studies have found that adaptive automation initiated by EEG-based measures of engagement enhance performance compared to yoked-control groups [21, 22], indicating that automation shifts that respond to levels of operator engagement enhance performance better than shifts that are not related to operator engagement. Interestingly, these studies have also found that negative feedback (defined as a decrease in the level of automation when engagement is low) was better than positive feedback (defined as an increase in the level of automation when engagement was high). This implies that for supervisory control applications, it may be effective to manage operator engagement and performance by using EEGbased adaptive automation.

Another study used EEG to measure workload, and compared performance on the adaptive automation that was triggered when workload was high to fully manual performance. The researchers found that performance was better with the EEG-based adaptive automation, but at the cost of temporarily reduced situation awareness [23]. This indicates that EEGbased measures of workload may not be as good as EEG-based measures of engagement in balancing performance and situation awareness.

A study conducted by Lagu, Landry, and Yoo [19] compared performance using HRV as a triggering condition to performance-based triggering of adaptive automation. They found that performance was best using HRV as a triggering condition, indicating that HRV may be a better initiator than performance-based measures. This study also manipulated the reallocation strategy used by the adaptive automation. The allocation strategies used were, complete, partial, and partial transformation. Performance was best under conditions of full reallocation (defined as shifting the entire task to automation).
Few other studies have investigated the effect of reallocation strategy directly.

The advantage of physiological assessment is that it can be used measure operator state continuously without any intervention from the operator. Therefore, physiological assessment may be better than performance-based measures for supervisory control applications because it doesn't require frequent actions to be taken by the operator. One disadvantage of physiological assessment is that is highly intrusive. In order to assess physiological state, operators have to be hooked up to equipment which can be somewhat bulky, can limit operator movement, and may be uncomfortable or unnatural to wear. Though typical physiological assessment requires that the operator be seated in a relatively stationary position, an adaptive automation system using a mobile physiological assessment tool has been demonstrated [23].

Another concern with physiological assessment is that there are individual differences in physiological responses, meaning that the system would have to be carefully calibrated for each operator. Further, there is day-to-day variability of physiological responses within individuals [24]. Both of these factors may limit the practicality of using physiological assessment of operator state as a way to trigger adaptive automation in the AdvSMR domain.

\subsection{Modeling}

Another way to initiate adaptive automation is to use models. Models are typically defined based on predicted human-performance consequences of specific scenarios, evolutions, or sets of conditions. Based on the anticipated effect on human performance, each of the circumstances modeled would be assigned a set level of automation. These models and levels of automation would be determined during the design phase, meaning that performance or operator state would not need to be measured during operation. One study investigated adaptive automation based on a predefined model of workload and compared performance using the adaptive automation with performance using clumsy automation (increasing automation during the low workload conditions) and manual control. The researchers found that performance was best under adaptive automation with matched workload, i.e., increased automation as workload increased [25], indicating that model-based adaptive automation can enhance performance compared to clumsy automation. Another study, conducted by [26], used a similar method based on a model of expected workload and compared two different schemes of adaptive automation. One used primarily manual control and the other used primarily supervisory control. The researchers compared performance and situation awareness in each adaptive automation condition to fully manual and supervisory control conditions. Situation awareness was best in manual control, followed by the adaptive automation condition with primarily manual control. The 
conditions with primarily supervisory control produced the lowest situation awareness. Further, the researchers found that there was an immediate drop in situation awareness following a shift in adaptive automation. Though these results indicate that model-based adaptive automation may be superior to manual performance, the efficacy of this initiator compared to other initiators is unknown. By definition, model-based triggering is not necessarily sensitive to an operator's true workload during operation, therefore it may have a limited benefit to performance.

\section{CONCLUSIONS}

Adaptive automation may be an effective way to manage human performance in supervisory control situations such as AdvSMR control rooms, however research needs to address a number of issues before the applicability and design of adaptive automation can be determined for AdvSMR applications.

The first issue is that the existing research does not consistently favor a particular triggering condition for adaptive automation. Most studies that investigate adaptive automation have found that adaptive automation, regardless of the triggering condition, enhances performance. This makes it difficult to assess the differential effectiveness of triggering condition because each method has a least some empirical evidence indicating that it is effective. Further, there are very few studies that directly compare different triggering conditions. Most of the studies select one triggering condition and compare adaptive automation to static levels. The majority of studies that compare adaptive automation performance to performance using static automation typically use fully manual and fully automatic levels, but do not compare adaptive automation to intermediate levels of automation. In order to fully assess the utility of adaptive automation compared to static automation, future research needs to compare adaptive automation across a variety of levels of automation.

The use of physiological measures, particularly EEGbased measures of engagement, to trigger adaptive automation is promising. In several studies, superior performance was reported for adaptive automation using these methods. However, there is still some question as to whether it will be practical to employ these methods in a control room due to the intrusiveness of the technology. Research and development efforts need to address the intrusiveness and data analysis burden of using EEG in the control room before it is truly feasible as a triggering condition in AdvSMR controls rooms.

Another promising method for triggering adaptive automation is adaptable automation using hierarchical task delegation. Research needs to address how to define the levels of abstraction for AdvSMR in the task delegation interface.
Many of the studies reviewed did not measure all of the important aspects of performance in order to fully assess the effectiveness of adaptive automation. Adaptive automation is typically regarded as a way to achieve enhanced system performance of increased automation while also maintaining operator situation awareness. Many studies measure operator performance, but not situation awareness. Furthermore, in order to assess the effect adaptive automation may have on an operator's ability to regain manual control, measuring situation awareness may not be sufficient. Future research should investigate the effect of adaptive automation on an operator's ability to resume manual control in the event of an automation failure (or the ability to detect an automation failure).

\section{REFERENCES}

[1] Bainbridge, L., 1983, "Ironies of Automation," Automatica, 19(6), pp. 775-779.

[2] Parasuraman, R., \& Riley, V. A., 1997, "Humans and automation: Use, misuse, disuse, abuse," Human Factors, 39, pp. 230-253.

[3] Sheridan, T., \& Parasuraman, R., 2006, "Humanautomation interaction," Reviews of Human Factors and Ergonomics, 1, pp. 89-129.

[4] Parasuraman, R., Bahri, T., Deaton, J. E., Morrison, J. G., \& Barnes, M., 1992, Theory and design of adaptive automation in aviation systems. Catholic University of America, Washington DC, Cognitive Science Lab.

[5] O'Hara, J., Higgins, J., \& Pena, M., 2012, "Human Factors Engineering Aspects of Small Modular Reactor Design and Operations," NUREG/CR-7126, US Nuclear Regulatory Commission, Washington, DC.

[6] Sauer, J., Nickel, P., \& Wastell, D., 2013, “Designing automation for complex work environments under different levels of stress," Applied ergonomics, 44(1), pp. 119-127.

[7] Kaber, D. B., \& Riley, J. M., 1999, “Adaptive Automation of a Dynamic Control Task Based on Secondary Task Workload Measurement," International journal of cognitive ergonomics, 3(3), pp. 169-187.

[8] Kidwell, B., Calhoun, G. L., Ruff, H. A., \& Parasuraman, R., 2012, "Adaptable and adaptive automation for supervisory control of multiple autonomous vehicles," In Proceedings of the Human Factors and Ergonomics Society Annual Meeting, Boston, MA, SAGE Publications, 56(1), pp. 428-432.

[9] Miller, C. A., \& Parasuraman, R., 2007, "Designing for Flexible Interaction between Humans and Automation: Delegation Interfaces for Supervisory Control," Human Factors: The Journal of the Human Factors and Ergonomics Society, 49(1), pp. 57-75.

[10] Parasuraman, R., Galster, S., Squire, P., Furukawa, H., \& Miller, C., 2005, “A Flexible Delegation-Type Interface Enhances System Performance in Human Supervision of Multiple Robots: Empirical Studies with 
Roboflag," Systems, Man and Cybernetics, Part A: Systems and Humans, IEEE Transactions on, 35(4), pp. 481-493.

[11] Miller, C. A., Shaw, T., Emfield, A., Hamell, J., Parasuraman, R., \& Musliner, D., 2011, "Delegating to Automation Performance, Complacency and Bias Effects under Non-Optimal Condition," In Proceedings of the Human Factors and Ergonomics Society Annual Meeting, SAGE Publications, Las Vegas, NV, 55(1), pp. 95-99.

[12] Shaw, T., Emfield, A., Garcia, A., de Visser, E., Miller, C., Parasuraman, R., \& Fern, L. , 2010, "Evaluating the Benefits and Potential Costs of Automation Delegation for Supervisory Control of Multiple UAVs," In Proceedings of the Human Factors and Ergonomics Society Annual Meeting, ). SAGE Publications, San Francisco, CA, 54(19), pp. 1498-1502.

[13] Inagaki, T., 2003, "Adaptive Automation: Sharing and Trading of Control," Handbook of Cognitive Task Design, E. Hollnagel Ed., LEA, pp. 147-169.

[14] Moray, N., Inagaki, T., and Itoh, M., 2000, “Adaptive Automation, Trust, and Self-Confidence in Fault Management of Time-Critical Tasks.” Journal of Experimental Psychology: Applied, 6(1), pp. 44-58.

[15] Kaber, D. B., Wright, M. C., Prinzel, L. J., \& Clamann, M. P., 2005, "Adaptive Automation of Human-Machine System Information-Processing Functions," Human Factors: The Journal of the Human Factors and Ergonomics Society, 47(4), pp. 730-741.

[16] Kaber, D. B., Perry, C. M., Segall, N., McClernon, C. K., \& Prinzel III, L. J., 2006, "Situation Awareness Implications of Adaptive Automation for Information Processing in an Air Traffic Control-Related Task," International Journal of Industrial Ergonomics, 36(5), pp. 447-462.

[17] Calhoun, G. L., Ruff, H. A., Spriggs, S., \& Murray, C., 2012, "Tailored Performance-based Adaptive Levels of Automation," In Proceedings of the Human Factors and Ergonomics Society Annual Meeting, SAGE Publications, Boston, MA, 56(1), pp. 413-417.

[18] Parasuraman, R., Cosenzo, K. A., \& De Visser, E., 2009, "Adaptive automation for human supervision of multiple uninhabited vehicles: Effects on change detection, situation awareness, and mental workload," Military Psychology, 21(2), pp. 270.

[19] Lagu, A. V., Landry, S. J., and Yoo, H-S., 2013, “Adaptive Function Allocation Stabilization and a Comparison of Trigger Types and Adaptation Strategies," International Journal of Industrial Ergonomics, pp. 1-11.

[20] Scerbo, M. W., Freeman, F. G., Mikulka, P. J., Parasuraman, R., Di Nocera, F. and Prinzel, L. J., 2001, "The Efficacy of Psychophysiological Measures for Implementing Adaptive Technology," NASA TP-2001211018, NASA Langley Research Center, Hampton, VA.

[21] Prinzel III, L. J., Freeman, F. G., Scerbo, M. W., Mikulka, P. J., and Pope, A., T., 2003, "Effects of a

Psychophysiological System of Adaptive Automation on
Performance, Workload, and the Event-Related Potential P300 Component," Human Factors: The Journal of the Human Factors and Ergonomic Society, 45(4), pp 601-604.

[22] Freeman, F. G., Mikulka, P. J., Scerbo, M. W., and Scott, L., 2004, "An evaluation of an adaptive automation system using a cognitive vigilance task," Biological Psychology, 67, pp. 283-297.

[23] Dorneich, M. C., Verves, P. M., Withlow, S.D., Mathan, S., Carciofini, J., and Reusser, T., 2006, "Neuro-

Physiologically-Driven Adaptive Automation to Improve Decision Making Under stress," In Proceedings of the Human Factors and Ergonomic Society Annual Meeting, SAGE Publications, San Francisco, CA, 50(3) pp. 410414).

[24] Christensen, J. C., Estepp, J. R., Wilson, G. F., \& Russell, C. A., 2012, "The Effects of Day-To-Day Variability of Physiological Data on Operator Functional State Classification," NeuroImage, 59(1), pp. 57-63.

[25] Parasuraman, R., Mouloua, M., \& Hilburn, B., 1999, "Adaptive Aiding and Adaptive Task Allocation Enhance Human-Machine Interaction," Automation technology and human performance: Current research and trends, pp. 119123.

[26] Kaber, D. B., Wright, M. C., \& Sheik-Nainar, M. A., 2006, "Investigation of multi-modal interface features for adaptive automation of a human-robot system," International journal of human-computer studies, 64(6), pp. 527-540. 